Nephrologe 2014 · 9:347-349

DOI 10.1007/s11560-014-0871-3

Online publiziert: 11. August 2014

(c) Springer-Verlag Berlin Heidelberg 2014

\author{
H. Haller ${ }^{1}$ J. Hoyer ${ }^{2}$ \\ ${ }^{1}$ Klinik für Nieren- und Hochdruckkrankheiten, Medizinische Hochschule Hannover \\ ${ }^{2}$ Klinik für Innere Medizin, Schwerpunkt Nephrologie, \\ Universitätsklinikum Gießen und Marburg GmbH, Standort Marburg
}

\title{
Hypertonie
}

\section{Liebe Kolleginnen und Kollegen,}

Der Schwerpunkt dieser Ausgabe von Der Nephrologe ist die Hypertonie, mit Beiträgen zur Pathophysiologie, Diagnostik und Therapie der Erkrankung. Das Gebiet der Hypertonie durchläuft zurzeit eine schwierige Phase. Nach Jahrzehnten der kontinuierlichen Entwicklung von neuen Medikamenten zur Behandlung dieser Erkrankung ist in den letzten Jahren auf diesem Gebiet eine deutliche Stagnation eingetreten. Wir verfügen heute über ein breites Arsenal von Medikamenten zur Behandlung dieser häufigen Erkrankung. Es scheint, dass auf dem therapeutischen Gebiet der sog. essenziellen Hypertonie keine großen Neuentwicklungen zu erwarten sind. Die pharmazeutische Industrie ist zurückhaltend und konzentriert sich im kardiovaskulären Bereich auf die Folgeerkrankungen des Hypertonus im kardiovaskulären System. Andererseits ist auf dem Gebiet der sog. resistenten Hypertonie, d. h. dort, wo medikamentös schwierig zu behandeln ist, eine rasante Zunahme von invasiven Verfahren zu beobachten. Und gerade auf diesem Gebiet der invasiven Verfahren, sowohl in der Behandlung der renovaskulären Hypertonie als auch in der Therapie der resistenten, essenziellen Hypertonie, ist eine heftige Diskussion entstanden. Unser Beitrag versucht hier, Klarheit zu schaffen.

Haller und Mitarbeiter versuchen nach der Veröffentlichung von Simplicity-3, den Standort von renaler Denervierung in der Therapie der Hypertonie neu festzulegen. Nach der initialen Begeisterung und den unkontrollierten überzeugenden Studiendaten ist durch die Veröffentlichung der großen randomisierten Studie eine heftige Diskussion über die Methode entbrannt. Nicht zuletzt die Diskussion über den Stellenwert randomisierter prospektiver Studien im Vergleich zu den sog. Registerdaten, welche in der täglichen Praxis erhoben worden sind, spielt hier eine Rolle. Wir versuchen, in diesem komplizierten Gebiet Orientierungshilfen zu geben.

Auch der Beitrag von Radermacher et al. beschäftigt sich mit einem viel diskutierten Thema. Die Nierenarterienstenose ist eine etablierte Diagnose, welche durch Dilatation bzw. Stenting behandelt wird. Dieses Verfahren wird in den vielen nephrologischen Zentren weltweit erfolgreich durchgeführt. Allerdings sind auch hier die publizierten randomisierten Studien eindeutig: Es ließ sich bislang kein Nachweis der Überlegenheit der invasiven Methode gegenüber der medikamentösen Behandlung führen. Die Veröffentlichung von CORAL hat hier nochmals deutlich gezeigt, wo die Limitationen der Methode liegen. Radermacher et. al. versuchen, auch hier Orientierungshilfen zu geben und ein rationales Vorgehen bei Patienten mit Nierenarterienstenose zu skizzieren.

\section{) Bislang ist eine Überlegenheit der invasiven Methode gegenüber der medikamentösen Behandlung der Nieren- arterienstenose nicht belegt}

Vor dem Hintergrund der großen Therapiestudien in den letzten Jahren sind die Leitlinien in der Hypertonie sowohl auf europäischer als auch auf internationaler Ebene neu ausgegeben worden. Zidek und Mitarbeiter kommentieren diese Leitli- nien kritisch. Da eine Überarbeitung der Leitlinien in den nächsten Jahren nicht zu erwarten ist, ist die jetzt vorliegende kritische Stellungnahme einer sehr erfahrenen Arbeitsgruppe auf dem Gebiet der Leitlinien in der Hypertonie von großer Bedeutung. Unser Kommentar versucht, auch hier Hinweise auf kritische Punkte in den Leitlinien und den pragmatischen Umgang damit zu geben.

Die Pathogenese der essenziellen Hypertonie ist immer noch ungeklärt. Unsere bekannten Vorstellungen über Salz- und Volumenregulation, die Exkretion von Natrium durch die Niere sowie die Wirkung von Kochsalz auf die Gefäße sind wichtige Komponenten in der Entstehung der essenziellen Hypertonie. Titze und Mitarbeiter haben diese Vorstellungen in den letzten Jahren erschüttert. Basierend auf den experimentellen Befunden, dass Kochsalzaufnahme nicht grundsätzlich mit einer Retention von Wasser verknüpft ist und es eine gewichtsunabhängige Einlagerung von Kochsalz gibt, haben sie ein neues pathophysiologisches Konzept der Kochsalzregulation entwickelt. Dies bedeutet auf der einen Seite, dass sich unsere Vorstellungen über die Regulation von Natrium und Chlorid im Körper ändern müssen. Zum anderen betrachtet die Arbeitsgruppe die Wirkung von Kochsalz auf die Entwicklung von Lymphgefäßen und damit von entzündlichen Erkrankungen in neuem Licht. Darüber hinaus hat sie Belege dafür, dass im menschlichen Körper sehr unterschiedliche Systeme an der Kochsalzregulation beteiligt sind. Ihre Arbeiten über diese "Rhythmen“ im menschlichen Körper sind aufsehenerregend und werden unsere Vorstellungen in den nächsten Jahren nochmals verändern. 
Wir hoffen, dass wir Ihnen mit diesen Themen ein interessantes Heft zusammengestellt haben, welches mit Interesse gelesen und für den praktischen Alltag von Bedeutung sein wird.

Mit freundlichen Grüßen

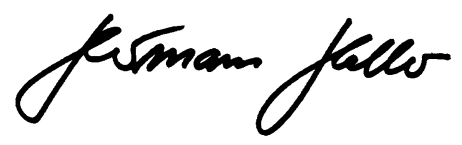

Hermann Haller

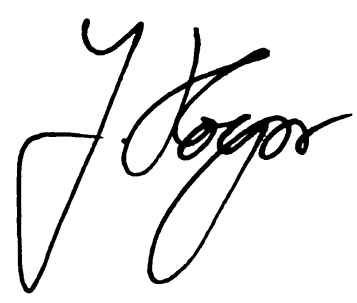

Joachim Hoyer

\section{Korrespondenzadressen}

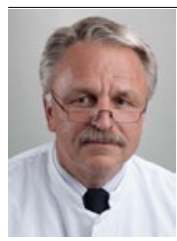

Prof. Dr. H. Haller

Klinik für Nieren- und

Hochdruckkrankheiten,

Medizinische Hochschule

Hannover

Carl-Neuberg-Straße 1,

30625 Hannover

haller.hermann@

mh-hannover.de

\section{Prof. Dr. J. Hoyer \\ Klinik für Innere Medizin, Schwerpunkt Nephrologie, Universitätsklinikum \\ Gießen und Marburg GmbH, \\ Standort Marburg \\ Baldingerstraße, \\ 35043 Marburg \\ hoyer@med.uni-marburg.de}

Interessenkonflikt. H. Haller und J. Hoyer geben an, dass kein Interessenkonflikt besteht.

\section{springermedizin.de - komfortabel recherchieren in der e.Bibliothek}

Ohne Umwege zur gewünschten Information zu gelangen - springermedizin.de macht's möglich. Verzichten Sie auf überquellende Stehsammler und unübersichtliche Papierstapel, und nutzen Sie statt dessen die digitale e.Bibliothek von Springer Medizin.

Suchen Sie Beiträge in einer bestimmten Fachzeitschrift Ihres Fachgebiets? Oder möchten Sie englischsprachige Journals für eine interdisziplinäre Recherche nutzen? Interessieren Sie sich für Übersichtsbeiträge oder aktuelle wissenschaftliche Studien?

Die e.Bibliothek wird all diesen Anforderungen gerecht: Sie umfasst über 600 deutschsprachige und internationale Fachzeitschriften aus allen Bereichen der Medizin inklusive der medizinischen Inhalte von SpringerLink.

Die e.Bibliothek beinhaltet auch „Online First"-Beiträge, also hoch aktuelle Beiträge, die bereits vor Erscheinen einer gedruckten Ausgabe in elektronischer Form publiziert wurden.

\section{Komfortable und schnelle Recherche}

- Mit der Volltextsuche von springermedizin.de durchsuchen Sie den gesamten Inhalt der e.Bibliothek und gelangen direkt zu den Inhalten, die für Sie relevant sind.

- AufWunsch können Sie die Suchergebnisse eingrenzen und beispielsweise gezielt in einzelnen Zeitschriften, nach Themen und sogar nach Autoren suchen.

- Wenn Sie einen englischen Suchbegriff eingeben, erhalten Sie zudem Treffer aus den englischsprachigen wissenschaftlichen Zeitschriften von SpringerLink.
Mit der Suchfunktion gelangen Sie auch zu Inhalten aus allen anderen Bereichen von springermedizin.de von zertifizierten Fortbildungskursen der e.Akademie, aktuellen Kongressberichten bis hin zu aktuellen Themendossiers, Videos und Nachrichten aus der Gesundheits- und Berufspolitk.

Ihre persönliche Merkliste

Finden Sie einen Beitrag besonders interessant oder möchten Sie ihn für die spätere Lektüre vormerken? Auf springermedizin.de können Sie ganz einfach Ihre persönliche Merkliste anlegen: ein Klick auf das Symbol "merken“ am Beitragsende genügt und die Beiträge erscheinen unter "Meine Merkliste".

Alle Beiträge sind als PDF-Datei im Layout der gedruckten Ausgabe sowie als HTML-Version verfügbar. In der HTMLVersion können Sie die Vorteile der verlinkten Literatur nutzen und direkt zu den zitierten Quellen gelangen.

e.Med - der Zugang zu allen digitalen Inhalten von Springer Medizin

Zugang zu allen Inhalten der e.Bibliothek bekommen Sie mit e.Med. Lernen Sie die Vorzüge dieses umfassenden Angebots kennen und testen Sie e.Med 30 Tage lang kostenlos und unverbindlich unter

\section{www.springermedizin.de/eMed}

Eine erfolgreiche Recherche wünscht Ihnen Ihr Redaktionsteam Fachzeitschriften von Springer Medizin 
Hier steht eine Anzeige.

黛 Springer 